\title{
Therapeutic Monoclonal Antibody Antagonizing Endothelin Receptor A for Pulmonary Arterial Hypertension ${ }^{\mathbb{S}}$
}

\author{
Cheng Zhang, Xiaofeng Wang, Hua Zhang, Chenjiang Yao, Hao Pan, Yong Guo, \\ Kesuo Fan, and Shuqian Jing
}

Gmax Biopharm LLC., Binjiang District, Hangzhou, Zhejiang, China

Received August 13, 2018; accepted April 9, 2019

\begin{abstract}
Endothelin receptor $A(E T A)$ is a G protein-coupled receptor and a major therapeutic target for pulmonary arterial hypertension (PAH). We took a novel approach and developed an antagonistic monoclonal antibody, getagozumab, specifically against ETA. Getagozumab displayed a $K_{d}$ value of $8.7 \mathrm{nM}$ and an $\mathrm{IC}_{50}$ value of $37.9 \mathrm{nM}$ in the cell-based assays. Getagozumab could significantly lower pulmonary arterial
\end{abstract}

pressure in both hypoxia-induced and monocrotaline (MCT)induced PAH monkey models and further attenuate the pulmonary arterial and right ventricular hypertrophy in MCTinduced PAH monkeys. The preclinical studies demonstrated that getagozumab is safe, long lasting, and efficacious. Getagozumab may provide a new and effective treatment for PAH patients.

\section{Introduction}

Pulmonary arterial hypertension (PAH) is a deadly disease marked by adverse remodeling of small pulmonary arteries and right ventricle and progressive pulmonary vascular constriction leading to right ventricular heart failure and death (Barst et al., 2004a,b; Lai et al., 2014). The vascular remodeling produced mainly by excessive cell proliferation causes the loss of the vascular luminal cross section to increase pulmonary vascular resistance (McLaughlin et al., 2009; Lau et al., 2017). The prevalence of PAH is about $15-50$ per million in the Western world (Humbert et al., 2006; Peacock et al., 2007), and in China it was estimated to be much higher (Zhai et al., 2010; Jiang and Jing, 2013).

Endothelin 1 (ET-1) is the most potent vasoconstrictor yet identified. ET-1 also acts as a growth-promoting factor of pulmonary artery vascular smooth muscle cells (Biasin et al., 2014) and a profibrotic factor (Hocher et al., 2000; Barton and Yanagisawa, 2008). The expression level of ET-1 is significantly upregulated in patients with PAH (Davenport et al., 2016). ET-1 is released by endothelial cells in relatively high amounts to act on endothelin receptor A (ETA) for the proliferation and constriction of vascular smooth muscle (Neylon, 1999; Davie et al., 2002). The ETA/ET-1 axis has been implicated in the pathogenesis of $\mathrm{PAH}$, and the other endothelin receptor, endothelin receptor B (ETB), mainly relaxes the vascular smooth muscle cells through a nitric oxide pathway

The research was funded by Gmax Biopharm LLC.

The authors were supported by Gmax Biopharm LLC.

https://doi.org/10.1124/jpet.118.252700.

S This article has supplemental material available at jpet.aspetjournals.org. and also acts on antiproliferation and ET-1 clearance (Hirata et al., 1993; Nelson et al., 2003).

Approved small molecular endothelin receptor antagonists have been beneficial to $\mathrm{PAH}$ patients in ameliorating disease symptoms through improving hemodynamics, exercise capability in 6-minute walk distance, and delaying time to clinical worsening (Rubin et al., 2002; Barst et al., 2004a,b; Galiè et al., 2008; Iglarz et al., 2008). Improvements in World Health Organization functional class, dyspnea, hemodynamics, and quality of life have also been observed in clinical studies along with substantial decreases in B-type natriuretic peptide following ambrisentan treatment (D'Alto, 2012; Macías SaintGerons et al., 2013; Maguire and Davenport, 2015). On the other hand, favorable pharmacokinetics/pharmacodynamics and toxicity as demonstrated by monoclonal antibody (MAb) may help to achieve efficacious therapeutics with a longer half-life (Deng et al., 2012; Zhao et al., 2012; Kamath, 2016), e.g., a once-a-month medication for PAH patients.

In this study, we generated antagonistic MAbs against ETA. We characterized the inhibitory activities of the MAbs in cell-based binding and functional assays. Furthermore, we demonstrated the in vivo efficacy of getagozumab in two PAH models of cynomolgus monkey. Our approach represents a new strategy for the treatment of PAH with a therapeutic antibody.

\section{Materials and Methods}

\section{In Vitro Pharmacology}

Cell Culture and Cell Lines. Stable cell lines expressing human, rat, or cynomolgus ETA were generated by transfection of an expression vector containing full-length human, rat, or macaque ETA cDNA into

ABBREVIATIONS: ET-1, endothelin 1; ETA, endothelin receptor A; ETB, endothelin receptor B; FACS, fluorescence-activated cell sorting; GPCR, G protein-coupled receptor; MAb, monoclonal antibody; MCT, monocrotaline; NOAEL, no observed adverse effect level; PAH, pulmonary arterial hypertension; PK, pharmacokinetics; RV, right ventricle; RVSP, right ventricular systolic pressure. 
CHO cells. Cells were maintained in DMEM/F12 (Invitrogen) supplemented with $10 \%$ dialyzed FBS minus hypoxanthine and thymidine at $37^{\circ} \mathrm{C}$, and the highly expressing cells were sorted by fluorescenceactivated cell sorting (FACS). Generally, cells were harvested using $10 \mathrm{mM}$ EDTA, counted, and resuspended in a suitable volume of the appropriate buffer for either FACS or assays.

Preparation and Generation of ETA MAbs and Getagozumab. The preparation and screening of monoclonal antibodies against ETA using hybridoma technology were conducted following the Gmax Biopharm antibody preparation platform and as described in Yan et al. (2009). The humanization was carried out via complementarity-depending region grafting as described previously (Li et al., 2018). The heavy and light chain variable sequences were independently aligned with the National Center for Biotechnology Information IgBLAST human germline database (https:// www.ncbi.nlm.nih.gov/igblast/), and the optimal germline sequences were selected as possible templates. In total, 613 mouse monoclonal antibodies with high-affinity binding toward human ETA were obtained, and the best antibody with antagonistic activity toward ETA was successfully humanized as an IgG4; i.e., getagozumab (also called GMA301).

Receptor Binding Assay. Getagozumab was labeled with DyLight 488 NHS Ester according to the manufacturer's instructions (Thermo Scientific). Getagozumab (100 nM) was incubated with the CHO cells stably expressing ETA of different species or the parental cells for 30 minutes at $4^{\circ} \mathrm{C}$ in PBS containing $2 \% \mathrm{FBS}$ and $0.02 \%$ sodium azide. Cell surface binding was quantified by FACS analysis, and data analysis was performed using Guava InCyte Software.

Calcium Influx Assay. Cells stably coexpressing ETA/aequorin were seeded in 96 -well cell culture plates $(35,000$ cells/well) and cultured overnight at $37^{\circ} \mathrm{C}$. The next day, the culture supernatant was removed, and coelenterazine (50 $\mu \mathrm{l}$; Promega) was added in the dark and incubated at $37^{\circ} \mathrm{C}$ for 2 hours. Then, $50 \mu \mathrm{l}$ of the hybridoma supernatant or a purified antibody (nine point, 1:3 dilution) was added and incubated at $37^{\circ} \mathrm{C}$ for 30 minutes. After incubation, $50 \mu \mathrm{l}$ of ET-1 at $20 \mathrm{nM}$ was injected and the flash luminescent signal was acquired in the time frame of 20 seconds with a SpectraMax L Microplate Reader (Molecular Devices). The $\mathrm{IC}_{50}$ values were calculated by nonlinear regression curve fitting using GraphPad Prism (GraphPad Software).

\section{In Vivo Pharmacology}

Animals. Normally, female or male cynomolgus monkeys (3 to 4 years old) were housed individually in cages in a laboratory animal facility under controlled temperature $\left(19-25^{\circ} \mathrm{C}\right)$, relative humidity (42\%-96\%), and a 12-hour light/dark cycle. The monkeys were fed twice a day with a regiment of $100 \mathrm{~g}$ diet plus $50 \mathrm{~g}$ of vegetables and fruit. The animals were given ad libitum access to water. Upon arrival, all animals were first quarantined for 19 days and the ones found free of diseases by physical checkup and biochemical analysis were included in the experiments.

Pharmacokinetics of Getagozumab in Cynomolgus Monkeys. The pharmacokinetics (PK) of getagozumab was determined in healthy cynomolgus monkeys by single intravenous injection. Blood samples were collected from each animal in 5,15 , and $50 \mathrm{mg} / \mathrm{kg}$ dose groups (number of animals in each group: $n=6$, three males and three females) at the 14 time points: preinjection; 1 minute; $0.5,2,4$, and 8 hours; and 1, 2, 3, 4, 7, 14,21, and 28 days. The samples were processed into plasma to determine the concentrations of getagozumab. The concentration of getagozumab was determined by ELISA using a captured antibody (6E8, an anti-getagozumab antibody generated in-house) that recognizes the antigen-binding fragment of getagozumab. The $\mathrm{GxH}$ IgG/horseradish peroxidase binding to the complex of 6E8getagozumab produced a chromogenic reaction in the presence of $3,3^{\prime}, 5,5^{\prime}$-tetramethylbenzidine (TMB) substrate. The absorbance was measured at the wavelength of $450 \mathrm{~nm}$, and the concentration of getagozumab was calculated by regression of the optical density value using the four parameter logistic model.
Toxicology Study of Getagozumab in Cynomolgus Monkeys. Single dose toxicity in cynomolgus monkeys was performed in dosing groups of 0 (formulation control), 75, 250, and $750 \mathrm{mg} / \mathrm{kg}$ by intravenous injection. Repeated dose toxicity in cynomolgus monkeys was performed in dosing groups (five females and five males per group) of 0 (formulation control), 25,75 , and $250 \mathrm{mg} / \mathrm{kg}$ by intravenous injection twice per week for 4 weeks, followed by a 6 -week recovery period. Similarly, repeated dose toxicity in cynomolgus monkeys was also performed in dosing groups (five females and five males per group) of $0,25,75$, and $250 \mathrm{mg} / \mathrm{kg}$ by intravenous injection once per week for 13 weeks, followed by a 6 -week recovery period.

Safety pharmacology endpoints were incorporated in the design of the repeated dose toxicity study. Blood pressure, heart rate, ECG, and body temperature were measured, and general status of the animals was observed and recorded.

In Vivo Study Using Hypoxia-Induced PAH Cynomolgus Model. Gmax Biopharm developed a hypoxia-induced cynomolgus PAH model together with CrownBio Inc (Taicang, China). The acute hypoxia-induced PAH model of cynomolgus monkey was induced by exposure to $12 \%$ hypoxia, and the pulmonary hypertension in monkeys was assessed quantitively using continuous-wave Doppler echocardiography (Lindqvist et al., 2011). At the same time, the heart rate and blood oxygen saturation were monitored as a safety measure. The same three monkeys were used for each treatment and the baseline was established prior to treatment. Ambrisentan was tested first, and after 1 week of washout getagozumab was tested.

The three monkeys were fasted overnight. After 2 to 3 hours of oral dosing of $1 \mathrm{mg} / \mathrm{kg}$ ambrisentan, they were anesthetized. Then, hypoxia was induced by $12 \%$ oxygen and the tricuspid regurgitation was measured at the same time. To determine if getagozumab attenuated hypoxia-induced $\mathrm{PAH}$ at $3,24,72,120$, and 168 hours after a single intravenous injection of $5 \mathrm{mg} / \mathrm{kg}$ getagozumab, the aforementioned experiments were repeated and performed sequentially at each time point.

In Vivo Study Using Monocrotaline-Induced PAH Cynomolgus Monkey Model. Gmax Biopharm developed a monocrotaline (MCT)-induced cynomolgus PAH model together with WuXi AppTech. The body weights of the animals ranged from 3 to $5.5 \mathrm{~kg}$ and the monkeys were divided into six groups of 6 , Sham (nonmodel formulation control), getagozumab $1.5 \mathrm{mg} / \mathrm{kg}$, getagozumab $5 \mathrm{mg} / \mathrm{kg}$, getagozumab $15 \mathrm{mg} / \mathrm{kg}$, MCT model (model formulation control), and ambrisentan oral $1 \mathrm{mg} / \mathrm{kg}$. The MCT-induced PAH cynomolgus model was established by injection of $30 \mathrm{mg} / \mathrm{ml} \mathrm{MCT}$ on days 1 and 14 . Getagozumab was administrated by intravenous injection on day 20 twice per week for 6 weeks.

Body weight and animal status were monitored throughout the study, and the blood routine and blood biochemical indicators (including alanine aminotransferase and aspartate aminotransferase) of all groups were measured before and after the drug administration.

Measurements of efficacy at the experimental endpoint included the following three parts. First, the animals were anesthetized with Zoletil ( $4 \mathrm{mg} / \mathrm{kg}$ dose), and the right ventricular systolic pressure (RVSP) was measured by insertion of a polyethylene catheter (outer diameter: $2.08 \mathrm{~mm}$ ) into the right jugular vein that was slowly advanced to the right ventricle. Second, the animals were euthanized according to animal euthanasia standard operating procedure, and then the heart, atria, and great vessels were removed. The left ventricle, right ventricle (RV), and septum were isolated; the left ventricle, $R V$, and septum were weighed after the water was blotted dry with filter paper. Using Fulton's index, RV hypertrophy was calculated as RV/(left ventricle + septum) $\times 100 \%$ (Giaid et al., 1993). Third, tissue slices of lung and RV were fixed with $10 \%$ neutral formalin, dehydrated, embedded in paraffin, sliced in paraffin, and hematoxylin-eosin-stained and mounted to measure the pulmonary arterial wall thickness (Zhou et al., 2015). The pulmonary arterial wall thickness is a ratio and is calculated by [(outer diameter - inner diameter)/2]/outer diameter of the vessel. Similarly, selected lung tissues were also actin stained to view the smooth muscle layer clearly (Behringer et al., 2016). 
The standards and guidelines for laboratory animals (GB149252001) (Ministry of Science and Technology, 2006a) established in China were strictly followed in the experiments. The studies of $\mathrm{PK} /$ toxicology, hypoxia-induced PAH, and MCT-induced PAH were conducted by JOINN Laboratory (Beijing, China), Crown Bioscience Inc. (Taicang, China), and WuXi App Tec, respectively. All three companies have earned Association for Assessment and Accreditation of Laboratory Animal Care international accreditation for their animal test facilities, and the experiment protocols were approved by their Institutional Animal Care and Use Committees beforehand.

\section{Results}

In Vitro Pharmacological Characterization of Antagonistic Antibodies Against Human ETA. In total, 613 murine monoclonal antibodies with specific binding to human ETA were identified. Multiple antibodies were potent antagonists of ETA and a few were humanized. Getagozumab was the most potent humanized antibody of IgG4 isotype.

The binding of getagozumab toward human ETA, human ETB, macaque ETA, and rat ETA was assessed by FACS analysis. The data in Fig. 1 demonstrate that getagozumab binds to human ETA specifically and only crossreacts with macaque ETA. Through saturation binding of human ETA, a $K_{\mathrm{d}}$ value of getagozumab was calculated as $8.7 \mathrm{nM}$ (Fig. $2 \mathrm{~A}$ ). The functional activity of getagozumab was studied in a calcium influx assay, where getagozumab effectively blocked calcium influx with an $\mathrm{IC}_{50}$ value of $37.9 \mathrm{nM}$ in human ETA-expressing CHO cells (Fig. 2B); however, it did not block calcium influx in human ETB-expressing CHO cells in comparison with the IgG control and dual receptor antagonist bosentan (Fig. 2C).

Pharmacokinetics of Getagozumab in Cynomolgus Monkeys after a Single Injection. Plasma concentrations of getagozumab were measured in the blood samples from the cynomolgus monkeys and subjected to ELISA assay. The PK profile of getagozumab is summarized in Table 1 and Fig. 3, and the conditions of the PK studies are summarized in the Pharmacokinetics of Getagozumab in Cynomolgus Monkeys section.

The concentration of getagozumab was found to increase with dose in a nonlinear manner, in which a 10 -fold increase of dose from 5 to $50 \mathrm{mg} / \mathrm{kg}$ resulted in 7.5-fold increase of exposure from 18.23 to 137.50 hour.mg/ml. The half-life of getagozumab after a single intravenous dose of 5,15 , or $50 \mathrm{mg} / \mathrm{kg}$ was approximately $9.90,7.21$, or 7.23 days in cynomolgus monkeys, respectively.

Toxicology Study of Getagozumab in Cynomolgus Monkeys. Single dose toxicity in cynomolgus monkeys was performed in dosing groups of $0,75,250$, and $750 \mathrm{mg} / \mathrm{kg}$, and the no observed adverse effect level (NOAEL) was $750 \mathrm{mg} / \mathrm{kg}$. In the single dose toxicity study, the animals were also evaluated by clinical observation, body weight, food intake, body temperature, clinical pathologic indicators of blood cell count, coagulation function, blood biochemical indicators, and urine analysis, in which there were no abnormalities observed. In addition, gross observation and histopathological examination were also performed, and no abnormal pathologic changes were observed in the gross anatomy examination of all animals in groups $1-4$.

Repeated dose toxicity in cynomolgus monkeys was performed in dosing groups of $0,25,75$, and $250 \mathrm{mg} / \mathrm{kg}$, and the NOAEL was $250 \mathrm{mg} / \mathrm{kg}$. The animals were treated by intravenous
A

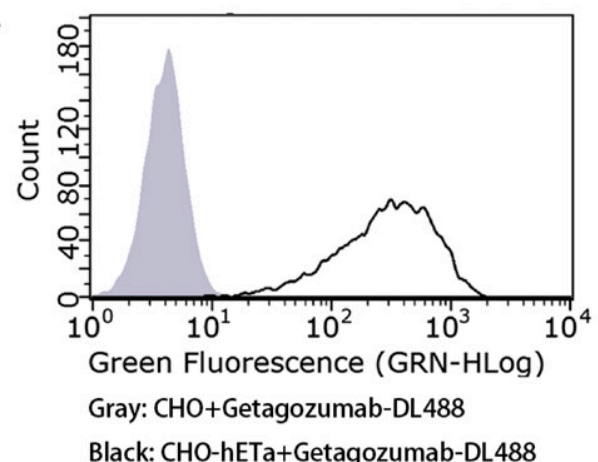

B

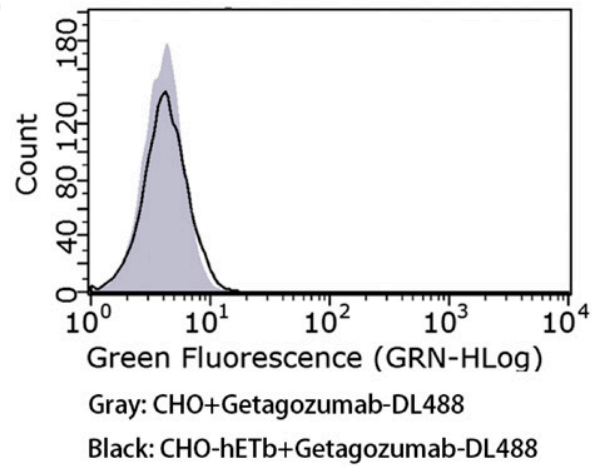

C

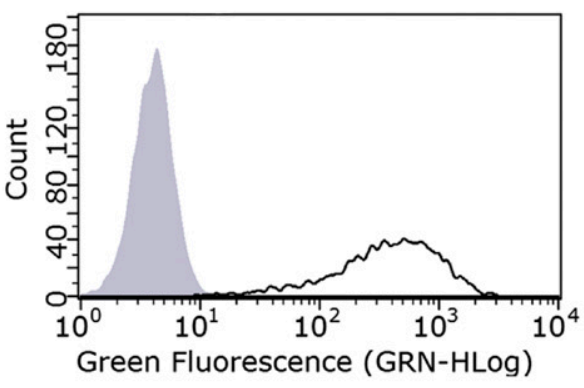

Gray: $\mathrm{CHO}+$ Getagozumab-DL488

Black:CHO-maETa+Getagozumab-DL488

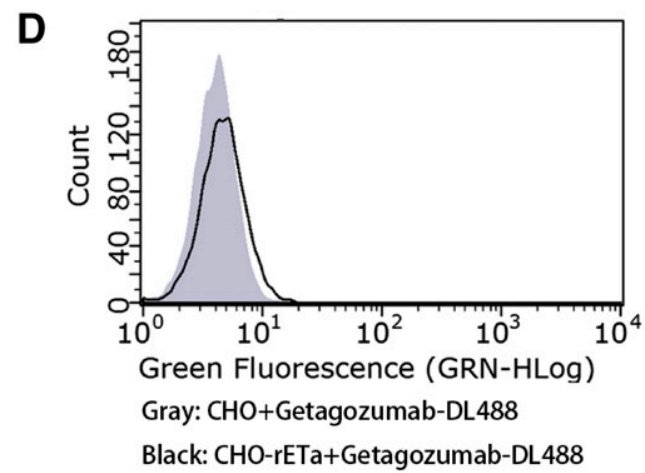

Fig. 1. (A-D) FACS analysis of $10 \mathrm{nM}$ of Dylight 488-labeled getagozumab binding to human ETA (hETA), human ETB (hETB), macaque ETA (maETA), and rat ETA (rETA)-expressing CHO cells.

injection twice per week for 4 weeks, followed by a 6 -week recovery period.

In the repeated dose toxicity study, no obvious toxicities were observed in heart rate, ECG, body temperature, and body weight. More importantly, the diastolic blood pressure/systolic blood pressure and alanine aminotransferase/aspartate 


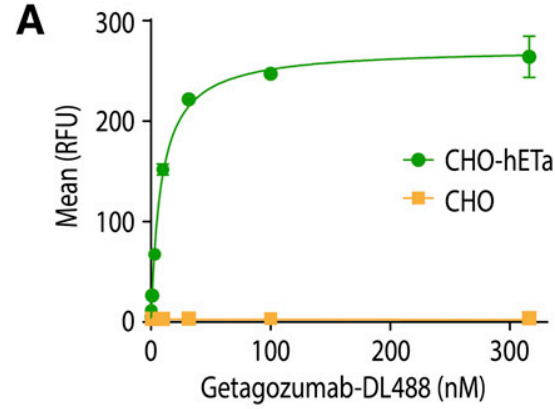

B
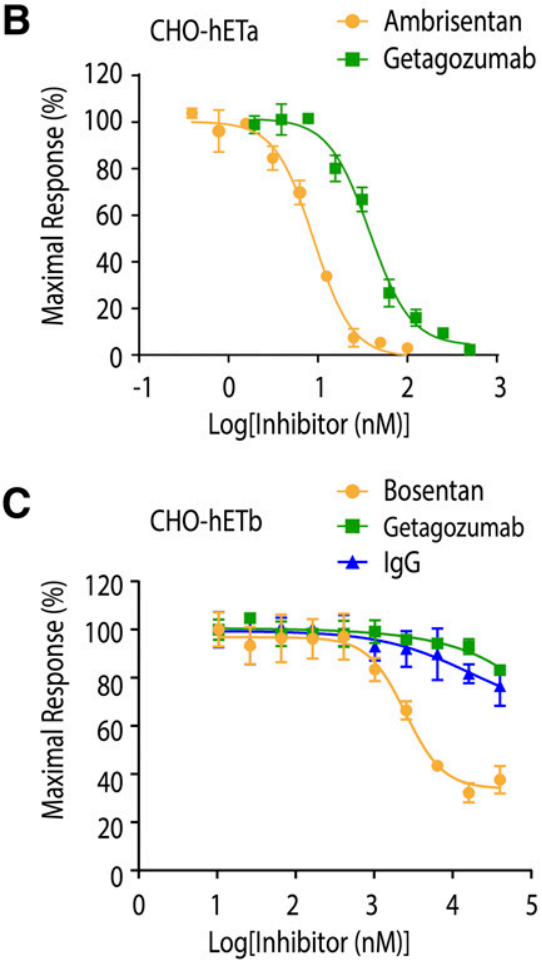

Fig. 2. (A) Saturation binding of Dylight 488-labeled getagozumab to CHO/dihydrofolate reductase/human ETA (hETA) cells (circles) and parental cells (squares). The geometric mean of fluorescence was calculated at each concentration of Dylight 488-labeled getagozumab, and the data were fitted with a one-site binding curve for the dissociation constant, $K_{\mathrm{d}}$. (B) Inhibition of ET-1-induced calcium influx by getagozumab and ambrisentan in hETA/aequorin coexpressing CHO cell line, with ET-1 at $20 \mathrm{nM}$. (C) Inhibition of ET-1-induced calcium influx by bosentan, but not by getagozumab in human ETB (hETb)/aequorin coexpressing CHO cell line, with ET-1 at $10 \mathrm{nM}$

aminotransferase in the 25,75 , and $250 \mathrm{mg} / \mathrm{kg}$ dose groups did not show any significant changes associated with getagozumab compared with the formulation control group (the $0 \mathrm{mg} / \mathrm{kg}$ group) (Supplemental Tables 1-3). Furthermore, no peripheral edema was observed in the monkeys of the three dosing groups or the formulation control group.

The red blood cells, hemoglobin, and hematocrit were decreased somewhat in the 4-week toxicity study of monkeys, while no red blood cells, hemoglobin, and hematocrit were decreased in the follow-up 13-week toxicity study of monkeys (dosing once per week). The data obtained for the red blood cells, hemoglobin, and hematocrit are provided in Supplemental Tables 6 and 7 . Therefore, there was no apparent sign of hemodilution. In addition, no abnormal results were observed in the bone marrow smear test and tissue histopathological examination (data not shown).
TABLE 1

The PK profile of getagozumab

Note: $\mu \mathrm{g} / \mathrm{ml}$ vs. $\mathrm{nM}$ in the table, $100 \mu \mathrm{g} / \mathrm{ml}=666.7 \mathrm{nM}$

\begin{tabular}{cccc}
\hline Dose & $T_{1 / 2}$ & $C_{\max }$ & AUC $_{\text {inf }}$ \\
\hline$m g / k g$ & days & $\mu g / m l$ & $h \cdot m g / m l$ \\
5 & $9.90 \pm 1.33$ & $98.22 \pm 16.81$ & $18.23 \pm 3.12$ \\
15 & $7.21 \pm 2.63$ & $317.57 \pm 38.19$ & $40.26 \pm 15.21$ \\
50 & $7.23 \pm 1.03$ & $1014.96 \pm 73.63$ & $137.50 \pm 23.34$ \\
\hline
\end{tabular}

$\mathrm{AUC}_{\text {inf }}$, area under the curve extrapolated to infinity; $T_{1 / 2}$, half-life.

For liver toxicity assessment, the liver enzyme data of the individual monkeys indicated no isolated case of liver injury (Supplemental Table 8).

Attenuation of PAH in a Hypoxia-Induced PAH Cynomolgus Model. The acute hypoxia-induced PAH cynomolgus monkey model was established by placing the animals under $12 \%$ hypoxia. Then, the antibody was administrated by intravenous injection at the $5 \mathrm{mg} / \mathrm{kg}$ dose. At 3, 24, 72,120 , and 128 hours after antibody administration, the tricuspid regurgitation velocity was monitored as described in the In Vivo Study Using Hypoxia-Induced PAH Cynomolgus Model section. The area under the curve of pulmonary arterial systolic pressure versus time was calculated; both getagozumab and ambrisentan showed a trend toward efficacy in reducing pulmonary arterial pressure, while getagozumab may be long lasting (up to 168 hours) (Fig. 4). Three monkeys were used for the experiment and the data on pulmonary arterial systolic pressure were not statistically significant. In Fig. 4, the yellow column represents the zero time point before drug treatment and the green columns represent the pulmonary arterial systolic pressure after drug administration.

Attenuation of PAH in an MCT-Induced PAH Cynomolgus Model. The MCT-induced PAH monkey model was established by two injections of MCT at the $30 \mathrm{mg} / \mathrm{kg}$ dose. Then, the animals were treated with getagozumab and ambrisentan. The efficacy of getagozumab at the $5 \mathrm{mg} / \mathrm{kg}$ intravenous dose twice per week was similar to ambrisentan at the $1 \mathrm{mg} / \mathrm{kg}$ intragastric dose once per day. After treating the $\mathrm{PAH}$ monkeys with getagozumab at the $15 \mathrm{mg} / \mathrm{kg}$ dose for 6 weeks, the RVSP was decreased by $54.0 \%$ compared with the MCT model group (Fig. 5A, $P<0.001$ ). The

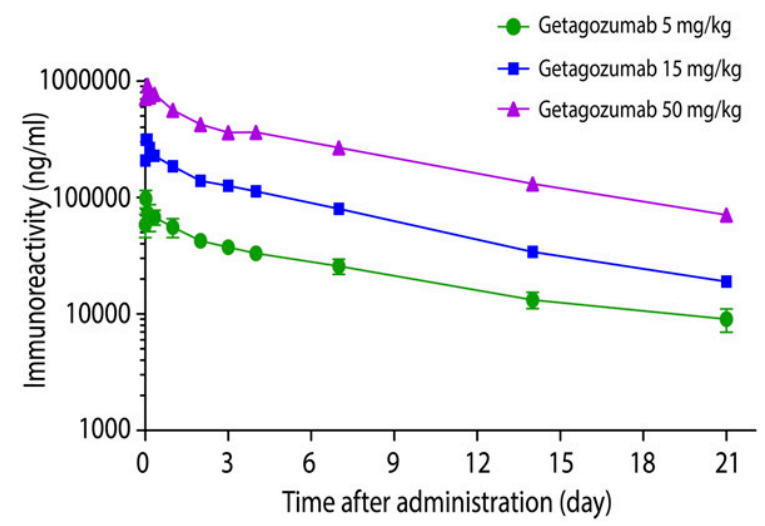

Fig. 3. Pharmacokinetics of getagozumab in cynomolgus monkeys. Getagozumab was administered as a single intravenous dose of 5, 15, or $50 \mathrm{mg} / \mathrm{kg}$; getagozumab in the blood was determined by sandwich ELISA. All data are expressed as mean \pm S.E.M., $n=6$. Note: $\mathrm{ng} / \mathrm{ml}$ vs. $\mathrm{nM}$ in the figure, $1000 \mathrm{ng} / \mathrm{ml}=6.67 \mathrm{nM}$. 


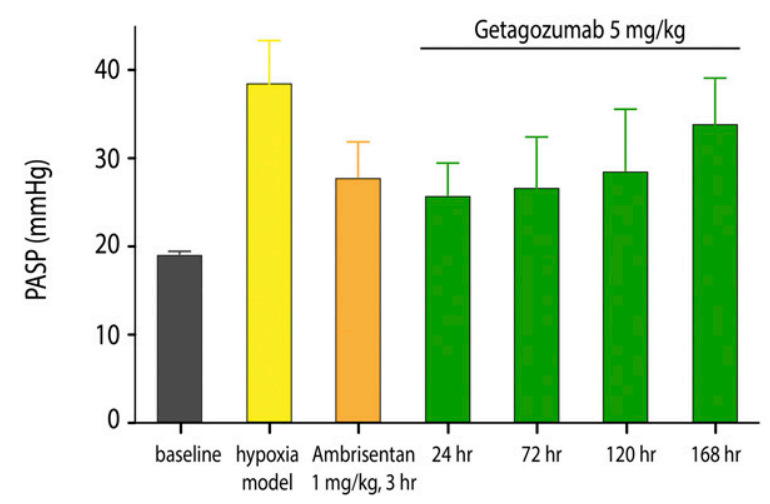

Fig. 4. Attenuation of pulmonary arterial systolic pressure (PASP) after a single intravenous administration of getagozumab at $5 \mathrm{mg} / \mathrm{kg}$ in the acute hypoxia-induced PAH cynomolgus monkeys. The yellow column represents the zero time point before drug treatment, and here the PASP before and after drug administration are compared.

percentage of inhibition was calculated by the equation $\left(1-\mathrm{RVSP}_{\text {getagozumab }} / \mathrm{RVSP}_{\text {model }}\right) \times 100 \%$ and the data from Table 2, specifically, $(1-19.3 / 42.0) \times 100 \%=54.0 \%$. The pulmonary arterial wall thickness and the right ventricular hypertrophy index (Fulton's index) (Ciuclan et al., 2011) were decreased by $47.8 \%$ and $38.7 \%$, respectively, compared with the MCT model group (Fig. 5, B and C, $P<0.001$ ), and the percentages inhibition were calculated similarly as previously described using data from Supplemental Table 5. Furthermore, getagozumab at the $15 \mathrm{mg} / \mathrm{kg}$ dose was significantly more efficacious than ambrisentan on RVSP $(P<0.017$, Table 2) at the $1 \mathrm{mg} / \mathrm{kg}$ intragastric dose once per day.

Figure 6 shows the results of the immunohistochemistry study of the pulmonary arteries by actin staining for the clear view of the smooth muscle layer. After treatment with getagozumab at the $15 \mathrm{mg} / \mathrm{kg}$ intravenous dose for 6 weeks, the getagozumab treatment significantly inhibited the thickening of the smooth muscle layer.

After day 1, there was no significant change in body weight with the administration of drugs, indicating that each group of drugs had no significant effect on body weight (Fig. 7). The blood and biochemical parameters (including alanine aminotransferase and aspartate aminotransferase) of the animals in each group had no significant changes before and after administration of getagozumab (Supplemental Table 4). During the experiment, no obvious abnormal changes were observed clinically, there were no deaths and near deaths in the animals, and their diet and spirit were fine.

In summary, as shown in Table 2 and Supplemental Table 5, getagozumab reduced the RVSP more than ambrisentan with statistical significance $(P<0.017)$ in the MCT-induced PAH cynomolgus monkeys. The other two responses of pulmonary arterial hypertrophy $(P<0.052)$ and right ventricular hypertrophy $(P<0.182)$ were trends but not statistically significant. The data were analyzed by one-way ANOVA and the least significant difference test.

For the $1.5 \mathrm{mg} / \mathrm{kg}$ dose group, blood sera were collected from the animals just before and after the first and 11th administrations; at 0 and 5 minutes; and at 2, 8, 24, 48, 72, and 96 hours. The serum concentrations of getagozumab were determined by ELISA for the following PK parameters: $C_{\max }=42.79 \pm 8.38$ and area under the curve extrapolated to infinity $=4.47 \pm 0.57$ for the first administration, and $C_{\max }=123.66 \pm 10.52 \mu \mathrm{g} / \mathrm{ml}$


Fig. 5. (A-C) Attenuation of the RVSP, pulmonary arterial wall thickness (PAWT), and right ventricular hypertrophy (Fulton's index) after intravenous injection of getagozumab twice a week for 6 weeks in the MCT-induced $\mathrm{PAH}$ cynomolgus monkeys. All data are presented as mean \pm S.E.M. and were analyzed statistically by one-way ANOVA plus Dunnett's $t$ test.

and area under the curve extrapolated to infinity $=21.19 \pm$ 11.01 hour $\cdot \mathrm{mg} / \mathrm{ml}$ for the 11 th administration. Note: $100 \mu \mathrm{g} / \mathrm{ml}=666.7 \mathrm{nM}$.

\section{Discussion}

G protein-coupled receptors (GPCRs) have been popular targets for small molecular drugs, and thus far there has not been much success in the development of antibody therapeutics targeting GPCRs (Hutchings et al., 2017). One of the main reasons is due to technical challenges in the search for functional antibodies of GPCRs. Here, we reported the generation and development of a MAb against ETA, a GPCR of family A, as a potential treatment of PAH.

The ET-1 expression level is upregulated in patients with $\mathrm{PAH}$, and the ETA receptor is also highly expressed in pulmonary arterial smooth muscle cells (Giaid et al., 1993). The ETA/ET-1 axis contributes to strong vasoconstriction 
TABLE 2

The RVSP in the efficacy study of the MCT-induced PAH monkeys

The data were analyzed by one-way ANOVA and least significant difference test.

\begin{tabular}{lcccccc}
\hline \multirow{2}{*}{ Cynomolgus } & Sham G1 & MCT Model G2 & Ambrisentan G3 & \multicolumn{3}{c}{ Getagozumab } \\
\cline { 5 - 7 } & & & & $1.5 \mathrm{mg} / \mathrm{kg} \mathrm{G} 4$ & $5 \mathrm{mg} / \mathrm{kg} \mathrm{G5}$ & $15 \mathrm{mg} / \mathrm{kg} \mathrm{G6}$ \\
\hline Cyno 1 & 17.4 & 40.3 & 21.7 & 34.0 & 20.5 & 18.2 \\
Cyno 2 & 13.8 & 43.0 & 20.2 & 42.2 & 25.0 & 17.5 \\
Cyno 3 & 15.4 & 39.8 & 32.4 & 19.6 & 22.7 & 20.3 \\
Cyno 4 & 16.3 & 40.6 & 23.6 & 37.6 & 22.0 & 19.2 \\
Cyno 5 & 22.6 & 43.7 & 28.4 & 41.4 & 29.7 & 17.0 \\
Cyno 6 & 16.4 & 44.3 & 26.3 & 36.3 & 22.8 & 19.3 \\
Mean & 17.0 & 42.0 & 5.2 & 8.9 & 4.3 & 1.3 \\
S.D. & 3.0 & 1.9 & & 0.001 & 0.215 & 0.017 \\
$P$ values vs. & 0.002 & 0.000004 & & & & \\
$\quad$ ambrisentan & & & & & & \\
\hline
\end{tabular}

Cyno, cynomolgus.

and smooth muscle cell proliferation and is pathophysiologically responsible for PAH (Barton and Yanagisawa, 2008). ETB receptors were also proposed to play a role in pathologic conditions (Yang et al., 2004), including PAH (Jasmin et al., 2001).

There were four small molecular drugs targeting the endothelin receptor, including bosentan, sitaxsentan, macitentan, and ambrisentan. These small molecular antagonists demonstrated improvements in pulmonary hemodynamics, exercise capacity, functional status, and clinical outcome in several randomized placebo-controlled clinical trials (Rubin et al., 2002; Barst et al., 2004a,b; Galiè et al., 2008; Iglarz et al., 2008), with adverse effects including elevation of liver transaminases, peripheral edema, anemia, and gastrointestinal reaction. Sitaxsentan had been approved for market in the European Union in 2006 (Humbert et al., 2014); However, Pfizer withdrew the drug from the market in 2010 due to several reports of fatal liver injury with the use of sitaxsentan in PAH patients (Barst et al., 2006). Bosentan and macitentan are dual endothelin receptor antagonists with ETA:ETB affinity ratios of $40: 1$ and $50: 1$, respectively (Dupuis and Hoeper, 2008; Monaco and Davila, 2016; Blok et al., 2017). Bosentan is associated with abnormal liver function as a common adverse effect in $10 \%$ of $\mathrm{PAH}$ patients, while macitentan is associated with a higher incidence of anemia. Ambrisentan is a relatively selective ETA blocker with an ETA:ETB affinity ratio of 77:1 and has demonstrated similar efficacy over bosentan and macitentan (Casserly and Klinger, 2009; Humbert et al., 2014).

Theoretically, selective small molecular antagonists of ETA should be more effective than nonselective small molecular antagonists, given the role played by ETB in both vasodilation and ET-1 clearance (Seferian and Simonneau, 2013). There is uncertainty as to whether small molecular endothelin receptor antagonists reduce mortality in $\mathrm{PAH}$ patients since the evidence that the small molecular endothelin receptor antagonists have an impact on the risk of death may be limited by the short duration of the studies and infrequent occurrence of this event in some trials (Liu et al., 2013; Monaco and Davila, 2016; Vaidya et al., 2017).

In this report, we undertook a novel approach by generating getagozumab, an antagonistic MAb targeting human ETA. This is the first report of such an inhibitory MAb of ETA. Getagozumab is not only safe, in general, but is also truly selective toward ETA. Getagozumab was found to have no side effects of liver toxicity and edema in the preclinical studies

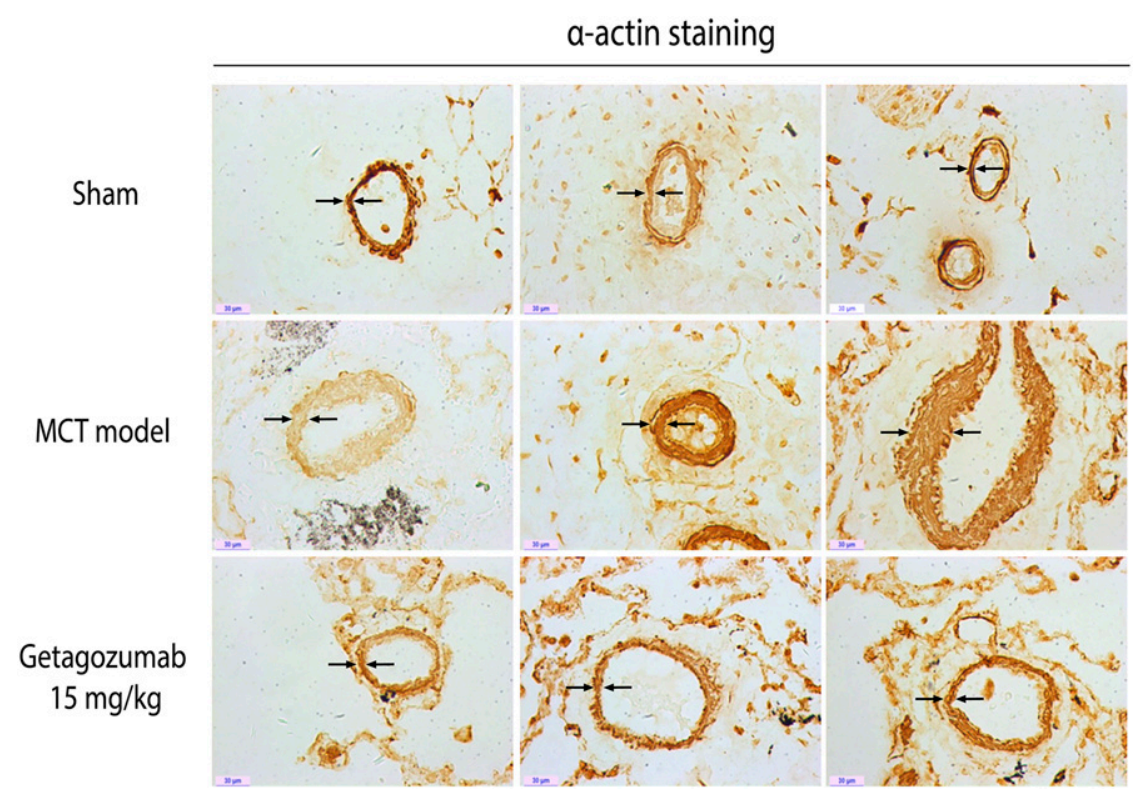

Fig. 6. Attenuation of pulmonary arterial hypertrophy in MCT-induced PAH cynomolgus monkeys was reflected in the $\alpha$-actin staining of the smooth muscle layer of the lung tissue slices. The magnification for each image is 200 -fold. 


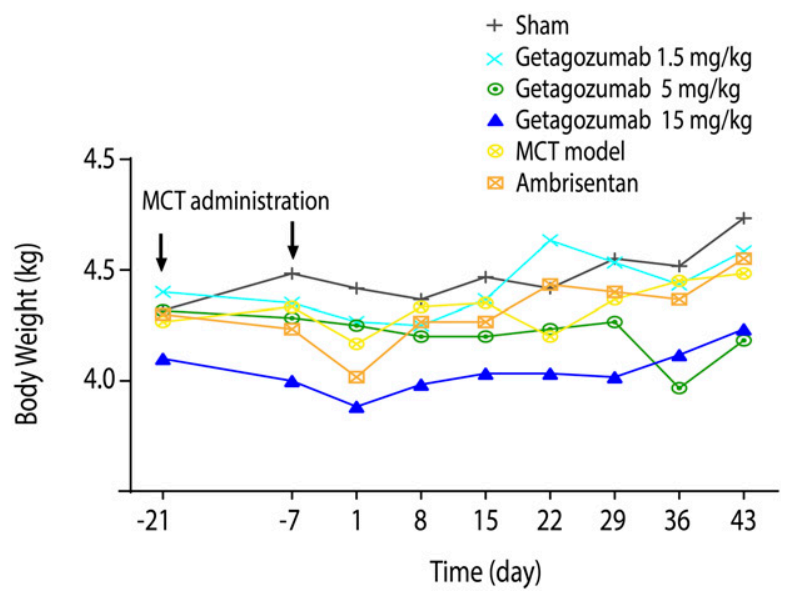

Fig. 7. Body weight before and after dosing of getagozumab.

with cynomolgus monkeys. Getagozumab binds to monkey and human ETA specifically. The in vitro functional activity of getagozumab was studied in a cell-based calcium influx assay with an $\mathrm{IC}_{50}$ value of $37.9 \mathrm{nM}$.

The PK study of getagozumab in cynomolgus monkeys revealed that at single intravenous doses of 5,15 , or $50 \mathrm{mg} / \mathrm{kg}$, its half-life is in the range of 7.21-9.9 days, and thus getagozumab may serve as a once-a-month treatment of PAH patients.

Acute and chronic toxicity studies were also pursued with NOAEL of $750 \mathrm{mg} / \mathrm{kg}$ for single dose toxicity and NOAEL of $250 \mathrm{mg} / \mathrm{kg}$ for 4 -week toxicity. The $250 \mathrm{mg} / \mathrm{kg}$ dosage is 50 times higher than the intended human clinical dose. Getagozumab has a clean safety profile, and no abnormalities in body weight, blood pressure, food consumption, ECG, and clinical pathology tests were observed despite the high doses.

Hypoxia-induced rodent PAH and MCT-induced rat models have been commonly used for the study of PAH (Zieliński, 2005; Ruiter et al., 2013). Since getagozumab does not crossreact with mouse or rat ETA, the PAH monkey model is needed for efficacy assessment. The hypoxia-induced PAH monkey model was established by acutely giving $12 \%$ hypoxia, which resulted in the rise of pulmonary arterial pressure. The effect of getagozumab at the $5 \mathrm{mg} / \mathrm{kg}$ intravenous dose was similar to ambrisentan at the $1 \mathrm{mg} / \mathrm{kg}$ intragastric dose, which is equivalent to 2 -fold of its clinical dose. Getagozumab showed a trend toward efficacy in reducing pulmonary arterial pressure up to 168 hours (Fig. 4) with no statistical significance, and three monkeys were used in the experiment.

The MCT-induced PAH model was also established in cynomolgus monkeys to study the pathologic changes before and after treatment with getagozumab. In comparison with the MCT model group, right ventricular pressure, pulmonary arterial hypertrophy, and ventricular hypertrophy were decreased significantly $(P<0.001)$ by as much as $54.0 \%, 47.8 \%$, and $38.7 \%$ respectively, after intravenous injection of getagozumab at the $15 \mathrm{mg} / \mathrm{kg}$ dose twice per week for 6 weeks.

Furthermore, getagozumab at the $15 \mathrm{mg} / \mathrm{kg}$ dose was significantly more efficacious than ambrisentan on the RVSP, and in this study the $15 \mathrm{mg} / \mathrm{kg}$ dose of getagozumab clinically corresponded to a human dose of $300 \mathrm{mg}$. At such a dose with $\mathrm{PAH}$ cynomolgus monkeys, getagozumab suppressed the rise of the pulmonary pressure completely. Importantly, as derived from the repeated dose toxicity study, even higher therapeutic doses could still be applied safely.

In this paper, we reported the first antagonistic Mab targeting human ETA. The data of the preclinical studies demonstrated that getagozumab was long lasting and significantly effective in attenuating right ventricular pressure and pulmonary arterial and right ventricular hypertrophy. Getagozumab had a clean safety profile. The US Food and Drug Administration has granted orphan drug designation to getagozumab, and clinical studies are under way to establish getagozumab as a novel and efficacious therapy for PAH patients.

\section{Acknowledgments}

JOINN Laboratory (Beijing, China), Crown Bioscience Inc. (Taicang, China), and WuXi App Tec (Shanghai, China) are acknowledged for collaborative work.

\section{Authorship Contributions}

Participated in research design: C. Zhang, Guo, Fan, Jing. Conducted experiments: Wang, H. Zhang, Yao, Pan.

Performed data analysis: Wang, H. Zhang, Yao, Pan.

Wrote or contributed to the writing of the manuscript: C. Zhang.

\section{References}

Barst RJ, Langleben D, Badesch D, Frost A, Lawrence EC, Shapiro S, Naeije R, and Galie N; STRIDE-2 Study Group (2006) Treatment of pulmonary arterial hypertension with the selective endothelin-A receptor antagonist sitaxsentan. $J$ Am Coll Cardiol 47:2049-2056.

Barst RJ, Langleben D, Frost A, Horn EM, Oudiz R, Shapiro S, McLaughlin V, Hill N, Tapson VF, Robbins IM, et al.; STRIDE-1 Study Group (2004a) Sitaxsentan therapy for pulmonary arterial hypertension. Am J Respir Crit Care Med 169:441-447. Barst RJ, McGoon M, Torbicki A, Sitbon O, Krowka MJ, Olschewski H, and Gaine S (2004b) Diagnosis and differential assessment of pulmonary arterial hypertension. $J$ Am Coll Cardiol 43(Suppl):40S-47S.

Barton M and Yanagisawa M (2008) Endothelin: 20 years from discovery to therapy. Can J Physiol Pharmacol 86:485-498.

Behringer A, Trappiel M, Berghausen EM, Ten Freyhaus H, Wellnhofer E, Odenthal M, Blaschke F, Er F, Gassanov N, Rosenkranz S, et al. (2016) Pioglitazone alleviates cardiac and vascular remodelling and improves survival in monocrotaline induced pulmonary arterial hypertension. Naunyn Schmiedebergs Arch Pharmacol 389:369-379.

Biasin V, Chwalek K, Wilhelm J, Best J, Marsh LM, Ghanim B, Klepetko W, Fink L, Schermuly RT, Weissmann N, et al. (2014) Endothelin-1 driven proliferation of pulmonary arterial smooth muscle cells is c-fos dependent. Int J Biochem Cell Biol 54:137-148.

Blok IM, van Riel ACMJ, van Dijk APJ, Mulder BJM, and Bouma BJ (2017) From bosentan to macitentan for pulmonary arterial hypertension and adult congenital heart disease: further improvement? Int J Cardiol 227:51-52.

Casserly B and Klinger JR (2009) Ambrisentan for the treatment of pulmonary arterial hypertension. Drug Des Devel Ther 2:265-280.

Ciuclan L, Bonneau O, Hussey M, Duggan N, Holmes AM, Good R, Stringer R, Jones P, Morrell NW, Jarai G, et al. (2011) A novel murine model of severe pulmonary arterial hypertension. Am J Respir Crit Care Med 184:1171-1182

D'Alto M (2012) An update on the use of ambrisentan in pulmonary arterial hypertension. Ther Adv Respir Dis 6:331-343.

Davenport AP, Hyndman KA, Dhaun N, Southan C, Kohan DE, Pollock JS, Pollock DM, Webb DJ, and Maguire JJ (2016) Endothelin. Pharmacol Rev 68:357-418.

Davie N, Haleen SJ, Upton PD, Polak JM, Yacoub MH, Morrell NW, and Wharton J (2002) $\mathrm{ET}_{\mathrm{A}}$ and $\mathrm{ET}_{\mathrm{B}}$ receptors modulate the proliferation of human pulmonary artery smooth muscle cells. Am J Respir Crit Care Med 165:398-405.

Deng R, Jin F, Prabhu S, and Iyer S (2012) Monoclonal antibodies: what are the pharmacokinetic and pharmacodynamic considerations for drug development? Expert Opin Drug Metab Toxicol 8:141-160.

Dupuis J and Hoeper MM (2008) Endothelin receptor antagonists in pulmonary arterial hypertension. Eur Respir $J$ 31:407-415.

Galiè N, Olschewski H, Oudiz RJ, Torres F, Frost A, Ghofrani HA, Badesch DB, McGoon MD, McLaughlin VV, Roecker EB, et al.; Ambrisentan in Pulmonary Arterial Hypertension, Randomized, Double-Blind, Placebo-Controlled, Multicenter, Efficacy Studies (ARIES) Group (2008) Ambrisentan for the treatment of pulmonary arterial hypertension: results of the ambrisentan in pulmonary arterial hypertension, randomized, double-blind, placebo-controlled, multicenter, efficacy (ARIES) study 1 and 2. Circulation 117:3010-3019.

Giaid A, Yanagisawa M, Langleben D, Michel RP, Levy R, Shennib H, Kimura S, Masaki T, Duguid WP, and Stewart DJ (1993) Expression of endothelin-1 in the lungs of patients with pulmonary hypertension. N Engl J Med 328:1732-1739.

Hirata Y, Emori T, Eguchi S, Kanno K, Imai T, Ohta K, and Marumo F (1993) Endothelin receptor subtype B mediates synthesis of nitric oxide by cultured bovine endothelial cells. $J$ Clin Invest 91:1367-1373.

Hocher B, Schwarz A, Fagan KA, Thöne-Reineke C, El-Hag K, Kusserow H, Elitok S, Bauer C, Neumayer HH, Rodman DM, et al. (2000) Pulmonary fibrosis and 
chronic lung inflammation in ET-1 transgenic mice. Am J Respir Cell Mol Biol 23:19-26.

Humbert M, Lau EM, Montani D, Jaïs X, Sitbon O, and Simonneau G (2014) Advances in therapeutic interventions for patients with pulmonary arterial hypertension. Circulation 130:2189-2208.

Humbert M, Sitbon O, Chaouat A, Bertocchi M, Habib G, Gressin V, Yaici A, Weitzenblum E, Cordier JF, Chabot F, et al. (2006) Pulmonary arterial hypertension in France: results from a national registry. Am J Respir Crit Care Med 173: 1023-1030

Hutchings CJ, Koglin M, Olson WC, and Marshall FH (2017) Opportunities for therapeutic antibodies directed at G-protein-coupled receptors. Nat Rev Drug Discov 16:787-810.

Iglarz M, Binkert C, Morrison K, Fischli W, Gatfield J, Treiber A, Weller T, Bolli MH, Boss C, Buchmann S, et al. (2008) Pharmacology of macitentan, an orally active tissue-targeting dual endothelin receptor antagonist. J Pharmacol Exp Ther 327: 736-745.

Jasmin JF, Lucas M, Cernacek P, and Dupuis J (2001) Effectiveness of a nonselective $\mathrm{ET}_{\mathrm{A} / \mathrm{B}}$ and a selective $\mathrm{ET}_{\mathrm{A}}$ antagonist in rats with monocrotaline-induced pulmonary hypertension. Circulation 103:314-318.

Jiang X and Jing ZC (2013) Epidemiology of pulmonary arterial hypertension. Curr Hypertens Rep 15:638-649.

Kamath AV (2016) Translational pharmacokinetics and pharmacodynamics of monoclonal antibodies. Drug Discov Today Technol 21-22:75-83.

Lai YC, Potoka KC, Champion HC, Mora AL, and Gladwin MT (2014) Pulmonary arterial hypertension: the clinical syndrome. Circ Res 115:115-130.

Lau EMT, Giannoulatou E, Celermajer DS, and Humbert M (2017) Epidemiology and treatment of pulmonary arterial hypertension. Nat Rev Cardiol 14:603-614.

Li C, Yang M, Wang X, Zhang H, Yao C, Sun S, Liu Q, Pan H, Liu S, Huan Y, et al. (2018) Glutazumab, a novel long-lasting GLP-1/anti-GLP-1R antibody fusion protein, exerts anti-diabetic effects through targeting dual receptor binding sites. Biochem Pharmacol 150:46-53.

Lindqvist P, Söderberg S, Gonzalez MC, Tossavainen E, and Henein MY (2011) Echocardiography based estimation of pulmonary vascular resistance in patients with pulmonary hypertension: a simultaneous Doppler echocardiography and cardiac catheterization study. Eur J Echocardiogr 12:961-966.

Liu C, Chen J, Gao Y, Deng B, and Liu K (2013) Endothelin receptor antagonists for pulmonary arterial hypertension. Cochrane Database Syst Rev (2):CD004434.

Macías Saint-Gerons D, de la Fuente Honrubia C, Montero D, and Catalá-López F (2013) Endothelin receptor antagonists-induced hepatotoxicity. Intern Med $J$ 43: $609-610$.

Maguire JJ and Davenport AP (2015) Endothelin receptors and their antagonists. Semin Nephrol 35:125-136.

McLaughlin VV, Archer SL, Badesch DB, Barst RJ, Farber HW, Lindner JR, Mathier MA, McGoon MD, Park MH, Rosenson RS, et al.; American College of Cardiology Foundation/American Heart Association (2009) ACCF/AHA 2009 expert consensus document on pulmonary hypertension: a report of the American College of Cardiology Foundation Task Force on Expert Consensus Documents and the American Heart Association: developed in collaboration with the American College of Chest
Physicians, American Thoracic Society, Inc., and the Pulmonary Hypertension Association. Circulation 119:2250-2294.

Monaco TJ and Davila CD (2016) Safety, efficacy, and clinical utility of macitentan in the treatment of pulmonary arterial hypertension. Drug Des Devel Ther 10: 1675-1682.

Nelson J, Bagnato A, Battistini B, and Nisen P (2003) The endothelin axis: emerging role in cancer. Nat Rev Cancer 3:110-116.

Neylon CB (1999) Vascular biology of endothelin signal transduction. Clin Exp Pharmacol Physiol 26:149-153.

Peacock AJ, Murphy NF, McMurray JJ, Caballero L, and Stewart S (2007) An epidemiological study of pulmonary arterial hypertension. Eur Respir $J$ 30:104-109.

Rubin LJ, Badesch DB, Barst RJ, Galie N, Black CM, Keogh A, Pulido T, Frost A Roux S, Leconte I, et al. (2002) Bosentan therapy for pulmonary arterial hypertension [published correction appears in N Engl J Med (2002) 346:1258]. N Engl J Med 346:896-903.

Ruiter G, de Man FS, Schalij I, Sairras S, Grünberg K, Westerhof N, van der Laarse WJ, and Vonk-Noordegraaf A (2013) Reversibility of the monocrotaline pulmonary hypertension rat model. Eur Respir J 42:553-556.

Seferian A and Simonneau G (2013) Therapies for pulmonary arterial hypertension: where are we today, where do we go tomorrow? Eur Respir Rev 22:217-226.

Vaidya B, Pangallo M, Ruffenach G, Cunningham CM, Perron JC, Kolluru S, Eghbali $\mathrm{M}$, and Gupta V (2017) Advances in treatment of pulmonary arterial hypertension: patent review. Expert Opin Ther Pat 27:907-918.

Yan H, Gu W, Yang J, Bi V, Shen Y, Lee E, Winters KA, Komorowski R, Zhang C, Patel JJ, et al. (2009) Fully human monoclonal antibodies antagonizing the glucagon receptor improve glucose homeostasis in mice and monkeys. J Pharmacol Exp Ther 329:102-111.

Yang LL, Gros R, Kabir MG, Sadi A, Gotlieb AI, Husain M, and Stewart DJ (2004) Conditional cardiac overexpression of endothelin-1 induces inflammation and dilated cardiomyopathy in mice. Circulation 109:255-261.

Zhai Z, Wang J, Zhao L, Yuan JX, and Wang C (2010) Pulmonary hypertension in China: pulmonary vascular disease: the global perspective. Chest 137(Suppl): 69S-77S.

Zhao L, Shang EY, and Sahajwalla CG (2012) Application of pharmacokineticspharmacodynamics/clinical response modeling and simulation for biologics drug development. J Pharm Sci 101:4367-4382.

Zhou SJ, Li M, Zeng DX, Zhu ZM, Hu XW, Li YH, Wang R, and Sun GY (2015) Expression variations of connective tissue growth factor in pulmonary arteries from smokers with and without chronic obstructive pulmonary disease. Sci Rep $\mathbf{5}$ 8564

Zieliński J (2005) Effects of intermittent hypoxia on pulmonary haemodynamics: animal models versus studies in humans. Eur Respir $J$ 25:173-180.

Address correspondence to: Dr. Cheng Zhang, Gmax Biopharm LLC., 288 Qiuyi Road, Binjiang District, Hangzhou 310052, Zhejiang, China. E-mail: czhang@gmaxbiopharm.com 